\title{
Usefulness of Carotid ultrasonography as a Screening test for Coronary artery disease in Korean diabetic patients
}

\author{
In-Jin Cho ${ }^{1}$, Sei Hyun Baik ${ }^{2}$, Yoo-Chul Hwang ${ }^{1}$, Kyu Jeung Ahn ${ }^{1}$, Ho-Yeon Chung ${ }^{1}$, In-Kyung Jeong ${ }^{1}$ \\ ${ }^{1}$ Department of Endocrinology and Metabolism, Kyung Hee University Hospital at Gangdong, Republic of Korea, \\ ${ }^{2}$ Department of Endocrinology and Metabolism, Korea University Guro Hospital, Republic of Korea
}

\section{Introduction and Objectives}

Carotid intima-media thickness (IMT) has shown an independent predictive marker of coronary artery disease (CAD) and cardiovascular disease in many studies. We investigated the usefulness of carotid IMT as a screening test for CAD in Korean diabetic patients.

\section{Materials and Methods}

We conducted a retrospective study from June 2006 to December 2012 at Kyung Hee University Hospital at Gangdong on the type 2 diabetic patients without a history of cardiovascular disease who underwent carotid ultrasonography (US) and stress echocardiography (SE). Medical record, laboratory data, carotid US, SE, and coronary angiography results of total 218 patients were reviewed.

Carotid US abnormalities were defined as $\geq 1 \mathrm{~mm}$ intima-media thickness or plaque detection. Significant CAD was defined as the presence $\geq 50 \%$ luminal narrowing of $\geq 1$ epicardial arteries or their major branches. We assessed predictive value for CAD based on the results of carotid US or SE.

\section{Results}

We analyzed 218 patients of type 2 diabetes mellitus (mean age: $61.2 \pm$ 10.3 years, male: $56.4 \%$, female: $43.6 \%)$. 116 patients $(53.2 \%$ of 218 patients) had carotid US abnormalities and 34 patients $(15.6 \%)$ showed positive SE. Patients with carotid plaque were older $(65.4 \pm 9.5$ years vs. $56.7 \pm 9.3$ years, $p<0.001$ ), had longer duration of diabetes (11.8 years vs. 7.8 years, $p<0.001)$, and lower body mass index $(25.1 \pm 3.4 \mathrm{~kg} / \mathrm{m} 2 \mathrm{vs} .26 .4$ $\pm 3.6 \mathrm{~kg} / \mathrm{m2}, \mathrm{p}<0.001$ ) compared with patients with normal carotid US (Table 1). However, there was no significant difference in prevalence of positive SE between patients with carotid plaque and patients with normal carotid US $(15.5 \%$ vs. $15.7 \%, p=0.968$, Table 2$)$.

Table 1 - Baseline characteristics of the study subjects

\begin{tabular}{|c|c|c|c|c|}
\hline & All subjects & IMT (-) & IMT $(+)$ & \\
\hline & $N=218$ & $\mathrm{~N}=102[46.8 \%]$ & $\mathrm{N}=116[53.2 \%]$ & $P$-value \\
\hline Age (years, mean \pm SD) & $61.23 \pm 10.3$ & $56.7 \pm 9.3$ & $65.39 \pm 9.5$ & $<0.001$ \\
\hline Male, n [\%] & $123[56.4 \%]$ & $62[60.8 \%]$ & $61[52.6 \%]$ & NS \\
\hline $\begin{array}{l}\text { Past or } \\
\text { Current smoking, n [\%] }\end{array}$ & $\begin{array}{c}97 / 200 \\
{[48.5 \%]}\end{array}$ & $\begin{array}{c}\mathbf{5 1} / 94 \\
{[54.3 \%]}\end{array}$ & $\begin{array}{c}46 / 106 \\
{[43.4 \%]}\end{array}$ & NS \\
\hline $\begin{array}{l}\text { Diabetes duration } \\
\text { (years, mean) }\end{array}$ & $9.9[0.1 \sim 40]$ & $7.8[0.1 \sim 40]$ & $11.8[0.1 \sim 0.40]$ & $<0.001$ \\
\hline BMI $\left(\mathrm{kg} / \mathrm{m}^{2}\right.$, mean $\left.\pm \mathrm{SD}\right)$ & $25.7 \pm 3.6$ & $26.4 \pm 3.7$ & $25.1 \pm 3.4$ & 0.009 \\
\hline HbA1c $(\%$, mean \pm SD $)$ & $7.8 \pm 1.9$ & $7.6 \pm 1.6$ & $8.0 \pm 2.1$ & NS \\
\hline HTN, n [\%] & $187[85.8 \%]$ & $83[81.4 \%]$ & $104[89.7 \%]$ & NS \\
\hline Dyslipidemia, n [\%] & $167 / 191[87.4 \%]$ & 76/89 [85.4\%] & $91 / 102[89.2 \%]$ & NS \\
\hline SBP $(m m H g$, mean \pm SD $)$ & $132 \pm 16$ & $131 \pm 15$ & $134 \pm 17$ & NS \\
\hline $\mathrm{DBP}(\mathrm{mmHg}$, mean $\pm \mathrm{SD})$ & $78 \pm 11$ & $79 \pm 10$ & $77 \pm 12$ & NS \\
\hline $\mathrm{TC}(\mathrm{mg} / \mathrm{dL}$, mean $\pm \mathrm{SD})$ & $175.9 \pm 41.0$ & $172.6 \pm 34.9$ & $178.8 \pm 45.7$ & NS \\
\hline TG $(\mathrm{mg} / \mathrm{dL}$, mean $\pm \mathrm{SD})$ & $154.7 \pm 95.2$ & $149.8 \pm 91.2$ & $159.1 \pm 98.8$ & NS \\
\hline HDL $(\mathrm{mg} / \mathrm{dL}$, mean $\pm \mathrm{SD})$ & $44.2 \pm 11.4$ & $44.8 \pm 11.1$ & $43.6 \pm 11.8$ & NS \\
\hline LDL $(\mathrm{mg} / \mathrm{dL}$, mean $\pm \mathrm{SD})$ & $103.2 \pm 34.5$ & $101.7 \pm 33.0$ & $104.6 \pm 35.8$ & NS \\
\hline $\begin{array}{l}\text { Mean IMT } \\
(\mathrm{mm}, \text { mean } \pm \text { SD }) \\
\end{array}$ & $0.73 \pm 0.18$ & $0.66 \pm 0.12$ & $0.79 \pm 0.20$ & $<0.001$ \\
\hline
\end{tabular}

NS, not significant

Table 2 - Proportion of stress echocardiography abnormalities in study subjects

\begin{tabular}{lcccc} 
& All subjects & IMT (-) & IMT(+) & \\
\cline { 2 - 4 } & $\mathrm{N}=218$ & $\begin{array}{c}\mathrm{N}=102 \\
{[46.8 \%]}\end{array}$ & $\begin{array}{c}\mathrm{N}=116 \\
{[53.2 \%]}\end{array}$ & $P$-value \\
\hline Stress echocardiography $(+)$ & $34[15.6 \%]$ & $16[15.7 \%]$ & $18[15.5 \%]$ & 0.968 \\
\hline
\end{tabular}

In patients with positive $S E$, there was no difference in prevalence of significant CAD whether they had carotid plaque or not [Significant CAD; IMT (-) \& SE (+): $68.8 \%$ (11 of 16 patients), IMT (+) \& SE (+): 88.9\% (16 of 18 patients), Figure 1].

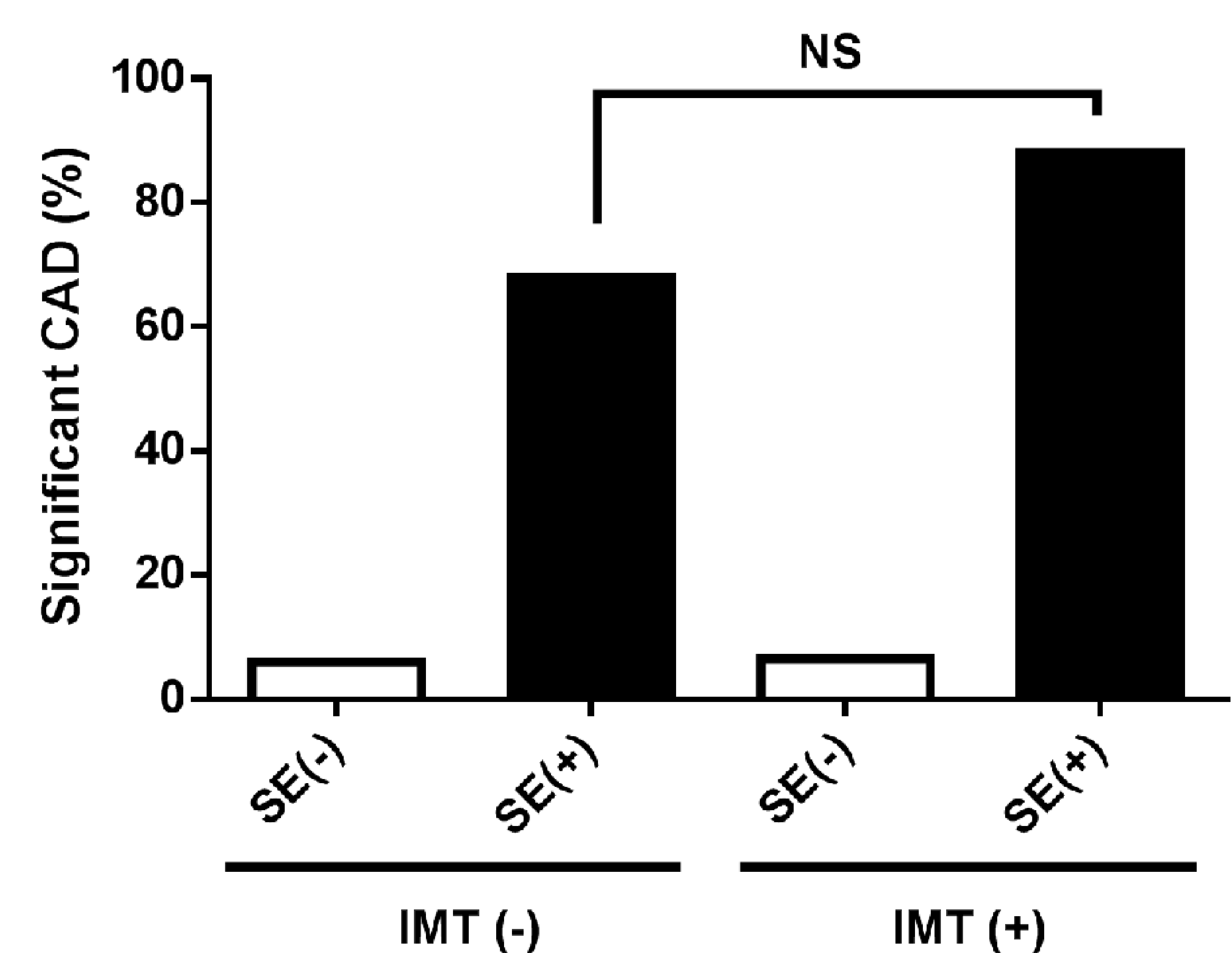

Figure 1- Prevalence of significant coronary artery disease (CAD) according to results of carotid intima-media thickness (IMT) and stress echocardiography (SE) (NS, not significant).

52 of 218 patients underwent coronary angiography. 32 of 52 patients had positive SE and positive predictive value of SE in significant CAD was $84.4 \%$ ( 27 of 32 patients, Table $3 A$ ). Of 32 patients with positive $S E, 16$ patients had carotid plaque and all of them had significant CAD (Table $3 B$ ).

Table 3 - Relationship between coronary angiography, stress echocardiography, and carotid intima-media thickness (IMT)

Table 3A

\begin{tabular}{|c|c|c|c|c|c|}
\hline \multicolumn{6}{|c|}{ Coronary angiography $(\mathrm{N}=52)$} \\
\hline \multicolumn{3}{|c|}{$\begin{array}{l}\text { Stress echocardiography }(-) \\
\quad \mathbf{N}=\mathbf{2 0}(38.5 \%)\end{array}$} & \multicolumn{3}{|c|}{$\begin{array}{c}\text { Stress echocardiography }(+) \\
\mathrm{N}=32(61.5 \%)\end{array}$} \\
\hline $\begin{array}{c}\text { Normal } \\
\mathrm{N}=4(\mathbf{2 0} \%)\end{array}$ & $\begin{array}{c}\text { Minimal } \\
\text { disease } \\
\mathbf{N}=\mathbf{5}(\mathbf{2 5 \%})\end{array}$ & $\begin{array}{c}\text { Significant } \\
\text { CAD } \\
\mathrm{N}=11(55.0 \%)\end{array}$ & $\begin{array}{c}\text { Normal } \\
\mathrm{N}=\mathbf{4}(12.5 \%)\end{array}$ & $\begin{array}{c}\text { Minimal } \\
\text { disease } \\
\mathbf{N}=\mathbf{1}(\mathbf{3 . 1 \%})\end{array}$ & $\begin{array}{c}\text { Significant } \\
\text { CAD } \\
\text { N=27 } \\
(84.4 \%)\end{array}$ \\
\hline
\end{tabular}

Table 3B

\begin{tabular}{|c|c|c|c|}
\hline \multicolumn{4}{|c|}{ Coronary angiography $(\mathrm{N}=52)$} \\
\hline \multicolumn{2}{|c|}{$\begin{array}{l}\text { Stress echocardiography }(-) \\
\mathbf{N}=\mathbf{2 0}(\mathbf{3 8 . 5 \% )} \\
\end{array}$} & \multicolumn{2}{|c|}{$\begin{array}{c}\text { Stress echocardiography }(+) \\
\mathrm{N}=32(61.5 \%)\end{array}$} \\
\hline $\begin{array}{c}\text { IMT }(-) \\
\text { N=7 (35.0\%) }\end{array}$ & $\begin{array}{c}\text { IMT (+) } \\
\text { N=13 (65.0\%) }\end{array}$ & $\begin{array}{c}\text { IMT (-) } \\
\mathrm{N}=16(50.0 \%)\end{array}$ & $\begin{array}{c}\text { IMT (+) } \\
\mathrm{N}=16(50.0 \%)\end{array}$ \\
\hline $\begin{array}{l}\text { Significant CAD } \\
N=5 \text { of } 7(71.4 \%)\end{array}$ & $\begin{array}{c}\text { Significant CAD } \\
\mathrm{N}=6 \text { of } 13(46.2 \%)\end{array}$ & $\begin{array}{c}\text { Significant CAD } \\
\mathrm{N}=11 \text { of } 16(68.8 \%)\end{array}$ & $\begin{array}{c}\text { Significant CAD } \\
\mathrm{N}=\mathbf{1 6} \text { of } 16(\mathbf{1 0 0 . 0 \% )})\end{array}$ \\
\hline
\end{tabular}

\section{Conclusions}

Carotid US abnormalities alone was not sufficient to draw SE results and to reliably predict CAD. However, when taken into carotid US results with SE results, they resulted in improving positive predictive value in significant CAD.

\section{References}

Ahmadvazir et al. Role of simultaneous carotid ultrasound in patients undergoing stress echocardiography for assessment of chest pain with no previous history of coronary artery disease. Am Heart J. 2014;168(2):229-36 Irie $\mathrm{Y}$ et al. The utility of carotid ultrasonography in identifying severe coronary artery disease in asymptomatic type 2 diabetic patients without history of coronary artery disease. Diabetes Care. 2013;36(5):1327-34 Lee et al. Non-invasive methods for cardiovascular risk assessment I asymptomatic type 2 diabetes mellitus. Korean Diabetes J. 2009;33:267-75.

Moon et al. The limitatio in measurement of carotid intima media thickness in type $\mathbf{2}$ diabetics with or without coronary artery disease. J Cardiovasc Ultrasound. 2008;16(4):116-22. 